\title{
El arte digital como estudio de caso de los problemas asociados a la conservación de documentos de archivo en el actual entorno tecnológico
}

\author{
Alejandro Delgado Gómez \\ Ayuntamiento de Cartagena (España)
}

\section{Resumen}

Desde un punto de vista tradicional, un documento de archivo es conservable porque tiene un contenido estable y una forma fija. Esta perspectiva ha sido modificada dramáticamente por un entorno digital caracterizado por la inestabilidad y la dinamicidad. Uno de los principales problemas de la archivística actual es definir los requisitos de conservación de este tipo de documentos. De igual modo, deberíamos abordar el hecho de que no todos los documentos digitales son conservables, sino que, más bien, meramente podemos proporcionarles continuidad. Con el objetivo de explorar los conceptos de conservación digital y de continuidad digital, el presente texto esboza en primer lugar una visión general de las técnicas de conservación en uso o en progreso, agrupadas en tres categorías: normas y buenas prácticas; tecnologías; soportes y formatos. En segundo lugar, selecciona una muestra de objetos procedentes del arte digital, carente de procesos reglados y de métodos rigurosos de conservación. Esta selección asume la siguiente aseveración: las tecnologías utilizadas para generar objetos de arte digital son muy similares a las empleadas para generar documentos de administración y gobierno electrónicos, de tal manera que las soluciones aplicadas a un entorno también pueden aplicarse al otro. Para llevar a cabo la selección se utiliza el criterio de incorporar objetos progresivamente dinámicos, multimediales y propietarios. Se analizan las propiedades técnicas de estos objetos desde el punto de vista de su conservación auténtica. A la luz de las técnicas esbozadas, se decide acerca de la posibilidad de conservación de los mismos. En aquellos casos en los que la conservación no es posible, se utiliza el concepto de continuidad; es decir, si un objeto no es conservable en su forma actual, ¿se puede garantizar al menos que seguirá siendo utilizable de algún modo? Los resultados que se espera obtener del presente ejercicio son los siguientes: ciertos objetos, presumiblemente los más estáticos y menos propietarios, serán conservables con las técnicas actuales; a algunos otros, dotados de mayor dinamicidad, se les podrá proporcionar continuidad, incluso en una forma 
distinta a la que tienen en la actualidad. Por último, ciertos objetos, probablemente los más propietarios, no serán conservables ni tendrán continuidad. Se pretende llegar a una doble conclusión: por una parte, la archivística ha de asumir que no todos los objetos digitales son conservables, aunque a muchos de ellos se les podrá dotar de continuidad para que sigan siendo utilizables; por otra, en un entorno tecnológico difícil, esta continuidad debe venir dada por la aplicación de determinadas normas, buenas prácticas, soportes, formatos y tecnologías, de los que, a modo de recomendación, se espera poder definir un listado relevante.

Palabras clave: Arte digital. Objetos digitales. Conservación. Continuidad.

\section{Abstract}

From a traditional point of view, a record is preservable because it has a stable content and a fixed form. This perspective has been dramatically modified by a digital environment, whose main features are instability and dynamism. One of the main concerns for current archival science is the definition of the requirements to preserve this kind of records. In the same way, we should address the inability to preserve the whole of digital records; rather, we merely would be able to provide them with continuity. Our objective is to explore the concepts digital preservation and digital continuity. With this aim, this text, first of all, outlines an overview of techniques for digital preservation, both in use and in progress. These are grouped in the following way: standards and best practices; technologies; media and formats. Secondly, it selects a sample of objects, coming from digital art, an environment lacking in regulated processes and rigorous methods of preservation. This selection assumes the following assertion: technologies used to generate objects in digital art are very similar to technologies used to generate records for e-government and e-governance, in such a way that the findings applied to an environment can be applied to the other one as well. In order to carry out our choice we incorporate progressively dynamic, multimedia and proprietary objects. We analyze technical properties in these objects, from the point of view of their authentic preservation. In the light of the above outlined techniques, we make a decision about the possibility of their preservation. Where preservation is not possible, we use the concept of continuity, that is to say, if we are not able to preserve an object in its current form, are we able to guarantee, at least, that it will be still usable in some way? We foresee we will be able to obtain from this exercise the following results: some objects, presumably, those more static and less proprietary, will be preservable with the current techniques; we will be able to provide some others, possessing a higher level of dynamism, with continuity, even in a form different to their current one. Finally, some objects, probably those that are more proprietary, will not be preservable and will not have continuity. From this exercise we foresee we will obtain two conclusions: on the one hand, archival

Scire. 14 : 2 (jul.-dic. 2008) 65-85. ISSN 1135-3716. 
science must assume that the whole of digital objects is not preservable, although most of them will be provided with continuity, in order to ensure their usability; and, on the other, in a difficult technological environment, this continuity should be provided by the use of a number of standards, best practices, media, formats and technologies. We hope we will be able to provide a list of some relevant means, as a final recommendation.

Keywords: Digital art. Digital objects. Preservation. Continuity.

\section{Introducción}

Desde un punto de vista tradicional, un documento de archivo es conservable porque tiene un contenido estable y una forma fija. Esta perspectiva ha sido modificada dramáticamente por un entorno digital caracterizado por la inestabilidad y la dinamicidad. Uno de los principales problemas de la archivística actual es definir los requisitos de conservación de este tipo de documentos. De igual modo, se debería abordar el hecho de que no todos los documentos digitales son conservables, sino que, más bien, meramente se les puede proporcionar continuidad. Con el objetivo de explorar los conceptos de conservación digital y de continuidad digital, el presente texto esboza en primer lugar una visión general de los modos de conservación en uso o en progreso, agrupados en tres categorías: normas y buenas prácticas; tecnologías; soportes y formatos. En segundo lugar, selecciona una muestra de objetos procedentes del arte digital, carente de procesos reglados y de métodos rigurosos de conservación. Esta selección asume la siguiente aseveración: las tecnologías utilizadas para generar objetos de arte digital son muy similares a las empleadas para generar documentos de administración y gobierno electrónicos, de tal manera que las soluciones aplicadas a un entorno también pueden aplicarse al otro (Duranti, 2005a). Para llevar a cabo la selección se utiliza el criterio de incorporar objetos progresivamente dinámicos, multimediales y propietarios. Se analizan las propiedades técnicas de estos objetos, desde el punto de vista de su conservación auténtica. A la luz de las técnicas esbozadas, se decide acerca de la posibilidad de conservación de los mismos. En aquellos casos en los que la conservación no es posible, se utiliza el concepto de continuidad; es decir, si un objeto no es conservable en su forma actual, ¿se puede garantizar al menos que seguirá siendo utilizable de algún modo? Los resultados que se espera obtener del presente ejercicio son los siguientes: ciertos objetos, presumiblemente los más estáticos y menos propietarios, serán conservables con las técnicas actuales; a algunos otros, dotados de mayor dinamicidad, se les podrá proporcionar continuidad, incluso en una forma distinta a la que tienen actualmente. Por último, ciertos objetos, probablemente los más propietarios, no serán conservables ni tendrán continuidad. Se pretende llegar a una doble conclusión: por una parte, la archivística ha de asumir que no todos los objetos digitales son conservables, aunque a muchos de ellos se 
les podrá dotar de continuidad para que sigan siendo utilizables; por otra, en un entorno tecnológico difícil, esta continuidad debe venir dada por la aplicación de determinadas normas, buenas prácticas, soportes, formatos y tecnologías, de los que, a modo de recomendación, se espera poder definir un listado relevante.

\section{Definiciones}

Si como petición de principio asumimos que una obra de arte digital es un documento y, si pasa a tener valor permanente y pluralizarse bajo ciertas condiciones, también un documento de archivo, debe definirse en primer lugar este concepto. Convencionalmente, se entiende por documento de archivo un "documento realizado o recibido en el curso de una actividad práctica como instrumento o producto de tal actividad, y guardado para acción o referencia" (1). Es en este sentido en el que se utiliza en el presente texto.

El concepto de arte digital resulta más difícil de definir, siquiera por el hecho de que cada corriente colectiva o creador individual interpreta su trabajo de diferente manera. La definición de arte del Diccionario de la Real Academia indica que es una "manifestación de la actividad humana mediante la cual se expresa una visión personal y desinteresada que interpreta lo real o imaginado con recursos plásticos, lingüísticos o sonoros". De manera más enriquecedora, la Enciclopedia británica define arte como "un objeto o experiencia conscientemente creada mediante una expresión de destreza o imaginación”. En la misma línea, el diccionario Merriam-Webster la define como "el uso consciente de destreza e imaginación creativa especialmente en la producción de objetos estéticos; también: obras así producidas".

Por su parte, digital se define como "la representación de un proceso físico mediante valores discretos binarios" (InterPARES 2 Project).

De la combinación de ambas definiciones derivamos el sentido en el que en el presente texto se utiliza arte digital: un producto creativo, no necesariamente persistente (puede ser un objeto, pero también una experiencia), aunque sí con valor cultural duradero, cuyas instancias se encuentran en soporte no analógico, sino digital. De esta manera, una fotografía, un texto o una pieza de música creados por medios analógicos pero guardados y reproducidos por medios digitales son arte digital; mientras que si se crean por medios digitales pero, digamos, se imprimen sobre papel o se almacenan y reproducen en una cinta de vídeo o de casete, son arte analógico. Esta definición coincide razonablemente con el concepto de documento digital del Proyecto InterPARES.

Existen varias acepciones para el término conservación, todas ellas relevantes para el asunto que nos ocupa: "1. La disciplina profesional de protección de materiales minimizando el deterioro y el daño químicos y físicos para minimizar la 
pérdida de información y para extender la vida de la propiedad cultural. 2. El acto de proteger de daño, erosión, decadencia o destrucción, especialmente mediante tratamiento no invasivo. 3. Derecho: La obligación de proteger los documentos y otros materiales potencialmente relevantes en un litigio y sujetos a descubrimiento" (Pearce-Moses, 2005).

\section{El problema de la conservación}

El presente texto asume que el arte digital - tanto el proceso como sus productos- es patrimonio cultural y que, en cuanto archiveros, tenemos la obligación de conservarla para uso de generaciones futuras. En el entorno tecnológico en el que nos desenvolvemos, no obstante, esta asunción implica la necesidad de abordar cierto número de problemas.

En primer lugar, en el actual debate profesional no siempre se tiene el mismo concepto de documento de archivo. De acuerdo con la conceptualización tradicional, que es la que asume, digamos, el Proyecto InterPARES, un documento de archivo debe tener un contenido estable y una forma fija. Si no los tiene, se debe intentar estabilizarlo y fijarlo, y, si esto no es posible, entonces no es conservable como documento de archivo, aunque lo sea como algún otro tipo de entidad. Téngase en cuenta que el Proyecto InterPARES adopta una perspectiva fuertemente jurídica y se enfoca sobre el concepto de autenticidad. Pero esto no siempre funciona en el contexto del arte, ni ahora ni en el pasado, lo cual es especialmente evidente en las artes escénicas. Por ejemplo, una representación de La flauta mágica es por definición perecedera: se agota al finalizar la función, salvo que alguien la grabe mediante una cámara de vídeo. Sin embargo, la grabación no es el documento de archivo de la representación, sino, por seguir utilizando la terminología de InterPARES, una copia, autenticada por el consenso cultural, de esta representación. El documento de archivo de la representación de La flauta mágica es la representación misma, que a su vez es una copia de la primera representación que Mozart dirigió de su obra y de la que no ha quedado constancia. En el entorno digital, un juego interactivo tal y como los que diseña Nick Montfort se agota en cada sesión. Puede que las interacciones de los distintos jugadores con el servidor en diferentes clientes repartidos por todo el mundo queden registradas en las bitácoras de sus equipos, pero el documento de archivo sería el proceso de jugar una partida. Si tuviéramos que buscar una contrapartida en un marco jurídico-administrativo, un juego interactivo o una representación de La flauta mágica serían similares al proceso de pagar con una tarjeta de crédito o efectuar una autoliquidación.

De acuerdo con el Proyecto InterPARES 2, se debe intentar fijar de alguna manera el contenido y la forma de una representación escénica o de un juego interactivo si se quiere conservarlos como documentos de archivo. Sin embargo, la fijación a través de, digamos, un pantallazo o una grabación en vídeo pasaría por alto

Scire. 14 : 2 (jul.-dic. 2008) 65-85. ISSN 1135-3716. 
aspectos esenciales del documento de archivo mismo, como las experiencias del público o la aleatoriedad de la partida. Por seguir con el ejemplo de Mozart, incluso aunque se recogieran las experiencias del público — de hecho, así se hizo en la versión que de La flauta mágica realizó Ingmar Bergman-, este documento de archivo estaría falseado, en la medida en que recogería estas experiencias de acuerdo con el punto de vista de Ingmar Bergman, no en términos objetivos, aunque la película del director sueco sería un documento de archivo auténtico en los archivos Bergman.

Un segundo argumento que utiliza el Proyecto InterPARES es la recomendación a los creadores de los procedimientos que tienen que seguir para que sus obras sean conservables de manera auténtica como documentos de archivo. Sin embargo, por decirlo en términos llanos, no se le pueden poner puertas al campo. En el marco de un sistema jurídico-administrativo, es necesario que el archivero intervenga sobre el sistema del creador con el fin de garantizar que se cumplen las condiciones para que los documentos de archivo que genera el creador sean buenos documentos desde el punto de vista técnico; es decir, el archivero debe hacer todo lo posible para que los documentos de archivo que se generen en un sistema jurídicoadministrativo sean auténticos, conservables y utilizables (2). Sin embargo, no es posible obligar a un creador de arte a adoptar una determinada tecnología o unos determinados procedimientos, que permitirán que su obra se conserve de manera auténtica pero al mismo tiempo condicionarán su opción estética. Incluso existen creadores, como sugiere McKemmish en su artículo fundacional sobre los archivos personales, que simplemente no quieren que su obra se conserve (McKemmish, 1996). Franz Kafka es un ejemplo de ambigüedad al respecto: habiendo intentado dejar reflejo de su biografía en todos sus escritos, en el último momento tomó la decisión de destruirlos, aunque, como es bien sabido, Max Brod, afortunadamente, no respetó esta decisión. Por utilizar un ejemplo procedente del arte digital, Jim Andrews comentó hace algún tiempo en conversación privada al autor del presente texto el caso de un colega que no quería dejar constancia de sus obras, salvo en el modo en que pudieran influir en la mentalidad de quienes las contemplaran (3). Los teóricos del continuo de documentos dirían que este artista dejaba "traza", que también es una forma de documento.

Esto nos lleva a la definición del segundo problema. La conceptualización por parte del Proyecto InterPARES del documento de archivo como algo dotado de contenido estable y forma fija es impecable desde el punto de vista jurídicoadministrativo; a nuestro juicio, se debe intentar aplicar siempre que sea posible en este contexto jurídico-administrativo, y contribuye a garantizar la autenticidad. Sin embargo, en un marco tecnológico crecientemente distribuido, interactivo y dinámico, esto solo es viable para ciertos tipos de documentos, aquellos similares a documentos analógicos, como un PDF o una base de datos relacional. Tal con- 
ceptualización no es aplicable a, digamos, un proceso (una representación escénica, un juego de ordenador) o una creación dinámica en lenguaje plano, como DHTML o XML en combinación con XSLT. Simplemente, no depende de nosotros: o es una entidad aleatoria, como el juego de ordenador, o se encuentra tan distribuida que no se pueden controlar todas las variables. Por ejemplo, si la dirección del URL donde se encuentra ubicado el esquema para XSLT cambia, las instancias de documento HTML resultantes de la hoja de estilo dejarán de funcionar.

En el presente texto se adopta la perspectiva de que, en un marco tecnológico crecientemente distribuido, interactivo y dinámico, y especialmente en el territorio de la creación artística, el concepto de documento de archivo debiera devenir más flexible. Muchas obras no tienen un contenido estable ni una forma fija: son documentos inestables, variables, repartidos entre diversos equipos, creados de acuerdo con diferentes tecnologías, de los que queda registro en una forma débil o falseada, o de los que no queda registro salvo en la forma de la traza que más arriba mencionábamos. Si es posible estabilizar su contenido y fijar su forma, sin forzar al creador a utilizar una tecnología o unos procedimientos limitativos y sin disminuir la riqueza de la obra, tanto mejor. Si no es posible, entonces debe conservarse a pesar de todo, llámese documento, información, datos, traza o lo que quiera que sea. Después de todo, Mozart no se planteó el problema de la conservación de sus obras, y no obstante las hemos conservado.

Otro problema que hemos anticipado es el de la libertad creadora. En condiciones ideales, los creadores utilizarían lenguajes abiertos, con posibilidades de migración futura, fácilmente accesibles, etcétera. Sin embargo, esto no siempre es así, ni podemos pretender que lo sea. Incluso aunque lo fuera, el uso de cualquier lenguaje o tecnología abiertos es problemático. Por ejemplo, cuando apareció DHTML, que es una combinación de lenguajes abiertos, Jim Andrews generó varias obras, como Animisms, por medio de este lenguaje, para PC y Mac. Sin embargo, puesto que Microsoft y Netscape introdujeron varias limitaciones propias, las versiones para Mac resultaron ilegibles al cabo de un tiempo. Andrews perdió la esperanza de que pudieran volver a reproducirse, hasta que un informático de una universidad finlandesa le envió por correo un código fuente modificado que permitía reproducir las versiones para Mac con la misma apariencia con las que Andrews las creó. Puesto que no se trataba del mismo código fuente, ni este había sido generado por el mismo autor, no eran la misma obra; sin embargo, Andrews, en tanto que creador, la reconoció como auténtica, y hoy día es perfectamente reproducible. Si esto sucede con una combinación de lenguajes abiertos, imaginemos lo que puede llegar a suceder con obras realizadas bajo lenguajes o programas propietarios, o necesitadas de plug-ins para su reproducción: applets de Java, flash, x3d, etcétera. De hecho, algunas obras creadas en los primeros tiempos de VRML, con plug-ins altamente inestables, son hoy en día irreproducibles. Finalmente, algunos 
creadores, como Ted Warnell o Jason Freeman, están generando obras a partir de programas o adaptaciones que ellos mismos han creado. En ambos casos los resultados se ofrecen en lenguajes abiertos (DHTML en el primero de ellos y mp3 en el segundo), pero el contexto de creación, si no se conservan los programas y la documentación acerca de ellos, puede quedar seriamente comprometido. Ciertamente, a efectos de conservación, de propiedad intelectual, de usabilidad, etcétera, los creadores debieran utilizar unas técnicas y procedimientos antes que otros; pero no podemos obligarlos. Así como en el caso de un sistema jurídico-administrativo el archivero debe participar activamente junto con el creador, en un sistema artístico la obligación de conservar tan auténticas como sea posible y por completo utilizables las obras corresponde al archivero.

A todo ello se añade el problema global por excelencia del actual entorno tecnológico: el problema de la obsolescencia del hardware, el software y los soportes, todos ellos con una esperanza de vida media mucho menor que los documentos que tienen que gestionar o guardar (ISO/TR 18492, 2005). Como ha mencionado con reiteración el autor del presente texto en otros lugares, ni siquiera en el caso de los lenguajes, formatos, soportes, etcétera, mejor acreditados en cuanto a sus posibilidades de futuro es posible garantizar este. Decir que un DVD o que XML y PDF tienen una vida media de cien años no significa que este DVD, este documento PDF o esta instancia XML durarán cien años, sino tan solo que ofrecen mayores posibilidades que otros soportes, formatos o lenguajes para abordar procesos de migración, conversión, emulación, etcétera, hacia soportes, formatos o lenguajes que todavía están por venir, en plazos cada vez más cortos. En estas condiciones, ¿qué cambios en la estructura, en el contenido, y en el contexto de los documentos habrá que realizar para que sigan siendo conservables y utilizables? ¿En qué momento tales cambios provocarán el que haya que declarar que el documento que se está conservando ya no es en absoluto el documento inicial? (4). A ello debe unirse la precariedad en la que se desenvuelven los objetos colocados en la Red. Si la dirección del URL o el servidor en que se encuentra alojada una obra desaparece, desaparece la obra misma, salvo que se tomen otras medidas de supervivencia. El proyecto Rhizome, por ejemplo, enlaza con la página donde se encuentra la obra original, pero también la clona en su propio servidor para prevenir la potencial pérdida del enlace.

En definitiva, diríase que, a la hora de abordar el problema de la conservación de arte digital, deberíamos diferenciar al menos dos aspectos esenciales: en primer lugar, cómo se han creado las obras, es decir, con qué software, utilizando qué lenguajes, bajo qué sistemas operativos, en qué soportes, etcétera. En segundo lugar, y a partir de este conocimiento previo, cuál es la mejor manera de conservar todo este conjunto de componentes que, unidos, dan forma a la obra. Es decir, en el caso de las obras de arte digital — como, por lo demás, en prácticamente todos los ám- 
bitos de conocimiento que utilizan nuevas tecnologías - se puede argumentar de manera concebible que la obra no es la última instancia o el resultado estable de un proceso, sino el proceso mismo y las herramientas que lo hacen posible, así como, más allá del momento en que el creador da por terminado el proceso, los procesos subsiguientes de interacción con los espectadores/usuarios.

\section{Modos de conservar}

La categorización de los modos de conservar en normas y buenas prácticas, tecnologías, y soportes y formatos es caprichosa, en la medida en que unos y otros se cruzan, interrelacionan y determinan entre sí. La única finalidad de esta categorización es facilitar el discurso al autor del presente texto y, deseablemente, la lectura al usuario del mismo.

\subsection{Normas y buenas prácticas}

Tal y como determinó el Proyecto InterPARES 1 (Duranti 2005b), el desarrollo de políticas, estrategias y normas es crucial para que la conservación se lleve a cabo con ciertas garantías de éxito. Organizaciones internacionales, cuerpos gubernativos y proyectos de investigación y desarrollo están realizando de unos años a esta parte significativos esfuerzos, de los que mencionaremos a modo de ejemplo unos pocos.

La Organización Internacional de Normalización (ISO), mediante varios de sus comités y subcomités, ha establecido un buen número de normas, en constante evolución, que contemplan diferentes aspectos de los problemas asociados a la conservación, especialmente desde el punto de vista técnico, aunque algunos informes han planteado la cuestión también en términos de desarrollo de políticas y estrategias (ISO/TR 18492, 2005). Sin embargo, gran parte de estas normas se han orientado hacia su uso en las organizaciones, no en el contexto del arte, o, más allá, de la cultura digital. De igual modo, muchas de ellas se han centrado en la necesidad de fijar los contenidos y, como esperamos poder extraer de algunos de los ejemplos de la siguiente sección, los documentos que nos ocupan rara vez pueden ser fijados. No obstante, algunos esfuerzos normativos, como la sanción de formatos abiertos o el desarrollo de normas de metadatos, resultan de utilidad al archivero que debe conservar obras de arte digital.

La prevención indicada en el párrafo anterior no significa que las normas y buenas prácticas orientadas a las organizaciones no sean de utilidad para conservar las obras de creadores individuales. Los tipos de documentos, las metodologías de conservación, las mejores tecnologías disponibles suelen ser iguales, y lo que cambian son los procesos - muy reglados en el caso de las organizaciones, muy abiertos en el caso de los creadores individuales- de creación. Excelentes ejemplos de las aportaciones que la conservación en organizaciones puede hacer a la conservación 
de obras de creadores individuales son Expertisecentrum David (eDavid) o Digital Longevity. A la inversa, el trabajo de conservación de obras de creadores individuales debería proporcionar aportaciones al trabajo de conservación en organizaciones.

En lo que se refiere a proyectos de buenas prácticas, las amplias bases de datos del proyecto Minerva, que recogen no solo ejemplos de buenas prácticas, sino también de centros de excelencia, y recomendaciones elaboradas desde diferentes puntos de vista, tendrían que poner de manifiesto el interés y la preocupación por resolver los problemas asociados a la conservación. En este marco, resultan de interés al área temática que nos ocupa, a modo de ejemplo, algunas de las guías publicadas por el Arts and Humanities Data Service. Fuera del ámbito del proyecto europeo, distintas organizaciones y centros de excelencia están investigando también en el problema.

\subsection{Tecnologías}

Ross Harvey ha llevado a cabo recientemente una excelente articulación de los problemas asociados a la conservación desde el punto de vista bibliotecario (Harvey, 2005, p. 101 y pássim). Dentro de su análisis, dedica una buena parte a los diferentes modos de conservación propuestos por gran parte de la literatura sobre el asunto. Este autor divide las propuestas tecnológicas de conservación en aquellas que sugieren conservar la tecnología (por ejemplo, la arqueología digital o la emulación) y aquellas que sugieren conservar el objeto (por ejemplo, la migración o el uso de formatos normalizados). En filigrana, Harvey define y estudia las siguientes posibilidades: las copias de seguridad analógicas (en papel, microfilm o formatos de imagen estática); la copia de seguridad digital; la copia de las secuencias de bits que constituyen el objeto; la canonización o traducción del objeto a formas canónicas; la arqueología digital, también conocida como recuperación de datos; los soportes durables o persistentes, la emulación, la encapsulación o creación de objetos persistentes; el almacenamiento masivo; la transferencia de soportes; el uso de metadatos; la migración, entendida como migración de formatos, normalización posterior a la migración, migración del software, migración de la versión y compatibilidad retrospectiva; desarrollo de políticas; refresco de los datos y del soporte; replicación, redundancia y conservación de múltiples copias; uso de formatos normalizados; conservación de la tecnología (por ejemplo, mediante museos tecnológicos o el uso de reproductores o visores obsoletos); conservación del código fuente; depósitos de software; recogida de dominios web; formalización o sustitución de los objetos por descripciones formalizadas de los mismos; extracción y estructuración de datos, etcétera.

De acuerdo con el punto de vista que hemos adoptado, se entiende que la aplicación de una o varias de estas tecnologías, en el área temática que nos ocupa, co-

Scire. 14 : 2 (jul.-dic. 2008) 65-85. ISSN 1135-3716. 
rrespondería al archivero, con independencia de que algunos creadores puedan ser más rigurosos que otros en lo que se refiere a la conservación de sus obras. Sin embargo, más allá de esta puntualización, lo que se desprende del anterior listado de tecnologías es el hecho de que, en el marco de los problemas asociados a la conservación, junto a los de tipo conceptual y técnico, uno de los más graves es el de los enormes costes asociados a la conservación de grandes volúmenes de objetos digitales (Harvey, 2005, p. 184; Vogt O'Connor, 2002). Una estrategia de evaluación, selección y eliminación, también con respecto a las obras de arte, será inevitable en el futuro, sobre la base de criterios que están por determinar. De igual modo, es necesaria la selección de las mejores tecnologías disponibles en función de aquello que se quiere conservar; y no solo de las mejores tecnologías disponibles, sino de las mejores tecnologías viables. De acuerdo con Kenneth Thibodeau (2002), una estrategia de conservación debe ser viable, sostenible, practicable y adecuada. De igual modo, el proyecto ERPANET (2003) ha preparado una pequeña guía para evaluar las mejores herramientas que pueden ser seleccionadas, atendiendo a diversos factores agrupados en cuatro criterios: generales (madurez, experiencia, diseminación, apertura, fiabilidad, modularidad y flexibilidad, costes), objetos (legislación, características, período de conservación), personas (destrezas, personal de mantenimiento, experiencia) y procesos (flujos de tareas, flexibilidad, buenas prácticas, requisitos de calidad).

En cualquier caso, y se adopte la aproximación que se adopte, no parece que se pueda pasar por alto el problema de los costes asociados a la conservación.

\subsection{Soportes y formatos}

Es una obviedad decir que no todos los soportes de almacenamiento tienen la misma longevidad ni son igualmente fiables; a pesar de ello, los soportes de almacenamiento son traidores. Por supuesto, a nadie se le ocurriría almacenar algo en un disquete de tres pulgadas y media; pero, incluso si se almacena en soporte CD-ROM o DVD, estos deben estar sometidos a rutinas de revisión y control de calidad para que la información que contienen no quede comprometida.

De igual modo, no todos los formatos ofrecen las mismas garantías de durabilidad. En líneas generales, aquellos que no poseen carácter propietario, que son abiertos o han sido sancionados por un organismo normalizador o un grupo de expertos son más fiables que los formatos propietarios o dependientes de elementos externos, como un reproductor comercial o libre. A modo de ejemplo, la guía NINCH aporta un exhaustivo listado de formatos digitales y una evaluación de aquellos que ofrecen mayor fiabilidad. Con todo, durante un proceso de evaluación y selección de formatos debe llegarse a un acuerdo entre durabilidad, finalidad prevista, espacio de almacenamiento necesario, componentes externos requeridos, estabilidad de estos componentes externos, etcétera. Por ejemplo, si la conservación

Scire. $14: 2$ (jul.-dic. 2008) 65-85. ISSN 1135-3716. 
a largo plazo de una imagen estática es la finalidad principal, entonces quizá se seleccione el formato TIFF y se almacenen los ficheros resultantes en un servidor y en soporte externo DVD, a pesar de que para la reproducción en web se precisará de un plug-in que el usuario final tenga que instalar en su equipo, algo que no tendría que hacer si se almacenaran como ficheros JPEG, que, adicionalmente, ocuparían menos espacio. Sin embargo, las posibilidades de tratar imágenes comprimidas en un momento posterior quedarían comprometidas.

Por último, debe mencionarse el creciente uso de XML, no solo como lenguaje de almacenamiento y conservación, sino también de transformación, comunicación y usos que todavía están por definir. En efecto, XML, en la medida en que es abierto, sencillo, interoperable y reutilizable, está siendo crecientemente adoptado en entornos archivísticos web para multitud de finalidades, bien en crudo, bien desarrollando esquemas o DTD para usos específicos. Resulta significativo que un grupo tan poco partidario de incorporar especificaciones abiertas como el Comité Técnico 171 de la Organización Internacional de Normalización esté utilizando XML para el desarrollo de su formato de intercambio de datos (ISO/DIS 22938, 2006). En lo que se refiere a la conservación de documentos archivísticos, distintos grupos de diversa procedencia han desarrollado modelos de metadatos de conservación que en otros lugares hemos mencionado (Delgado, 2005). De ellos, el que ha alcanzado mayor difusión quizá sea PREMIS, desarrollado por el Online Computer Library Center (OCLC) y el Research Libraries Group (RLG), que propone un enfoque integrador, independiente de la tecnología, utilizable en varias capas y para diversas entidades, y apoyado en XML.

Finalmente, si es cierto, como se viene sugiriendo últimamente (GillilandSwetland, 2005), que los metadatos y los lenguajes de descripción archivística satisfacen, o son susceptibles de satisfacer, simultáneamente más de una función, y que la descripción archivística es ya una forma de conservación, entonces los diferentes esfuerzos, custodiales y poscustodiales, por mejorar la descripción archivística y la asignación de metadatos constituyen igualmente un modo de garantizar que el contexto de los objetos se conserva, junto con los objetos mismos.

\section{Ejemplos}

Los siguientes ejemplos tienen por finalidad mostrar en la práctica algo de lo que llevamos dicho hasta el momento, así como dejar abierto el reto de intentar aplicar los procedimientos de conservación esbozados en la sección anterior a objetos difíciles. La selección de los ejemplos no es aleatoria; pretende ser progresivamente compleja, pero se limita, con un par de interesantes excepciones, a algunos de los artistas implicados en el proyecto de creación Jaguar puro inmarchito, destinado, entre otros muchos productos, a homenajear a la escritora española Carmen Conde en su centenario.

Scire. 14 : 2 (jul.-dic. 2008) 65-85. ISSN 1135-3716. 
Antes de proponer otros ejemplos, quisiéramos sugerir la revisión del clásico de Olia Lialina My boyfriend came back from the war. Como es bien sabido, Lialina realizó esta obra en 1996, por medio de frames de HTML. El aspecto del original, que vincula de manera aparentemente aleatoria texto e imágenes en blanco y negro, es algo rústico, pero, puesto que fue creado haciendo uso de un lenguaje abierto que los navegadores reconocen actualmente, se puede reproducir sin problemas. La sensación de infinitud que proporcionan los frames es engañosa; en realidad, toda la obra se apoya en un número limitado de ficheros fácilmente identificables y conservables, junto con sus vínculos. A partir del original, diversos autores han venido realizando varias versiones, por medio de diferentes tecnologías: HTML, más HTML pero con imágenes coloreadas, o con diferentes imágenes y sin texto, flash, creaciones analógicas (gouaches o camisetas comerciales) pasadas a soporte digital, realidad virtual para la que se precisa un plug-in prácticamente inencontrable, gifs animados, vídeo pasado de analógico a digital en diversos formatos más o menos abiertos (avi, rm, mpg), presentaciones en PowerPoint, aplicaciones de creación propia no reproducibles en la actualidad, críticas y versiones cuyos enlaces se han perdido, blogs... Una completa gama de las posibilidades y los peligros asociados con el arte digital en el actual entorno tecnológico.

Jim Andrews edita una excelente revista digital, Word For/Word, cuyos contenidos son poemas textuales creados generalmente en un procesador de textos y convertidos a HTML, o imágenes jpg o gif, o fotografías digitales de obras analógicas. En la medida en que utilizan especificaciones casi siempre abiertas y constituyen un solo fichero, a lo sumo un pequeño número controlable de ficheros, estas obras son fácilmente conservables y utilizables con las tecnologías actuales. Además, será presumiblemente fácil migrarlas a nuevas tecnologías. Sin embargo, la revista misma está realizada mediante flash, de tal modo que el acceso desde ella a las obras queda comprometido, puesto que los navegadores no leen bien flash.

La excelente poetisa digital argentina Ana María Uribe, ya fallecida, creó la mayoría de sus Tipoemas y Anipoemas mediante gifs animados anidados en HTML. Son por tanto previsiblemente fáciles de conservar y utilizar, aunque la técnica del gif animado ha devenido obsoleta y no existe ningún motivo para seguir sosteniéndola. Juega a su favor el hecho de que se trata de una técnica muy sencilla.

El mismo Jim Andrews y sus colaboradores cercanos han incorporado en el portal Vispo.com el uso de javascripts en algunas de sus obras, así como la creación de audio interactivo partiendo de flash, aunque Andrews ha desarrollado el software de creación propia Nio, y anteriormente Vismu, cuyo código fuente ha puesto a disposición de todo aquel que quiera utilizarlo. Mediante Vismu, y partiendo de unos pocos ficheros de sonido, el usuario final puede crear de manera interactiva música aleatoria. De igual modo, ha desarrollado Arteroids, un juego razonablemente similar a cualquiera juego de PlayStation en el que el jugador debe 
defender palabras que se atacan entre ellas. En estos casos, al problema de la durabilidad de la tecnología utilizada, así como de la conservación del software original y la documentación asociada a su uso, se une el de cómo de conservar las interacciones entre el jugador, ubicado en un cliente remoto, y el juego, ubicado en otro servidor remoto. $\mathrm{Si}$, como dijimos anteriormente, el documento es la partida misma, no hay modo de conservarlo. Una alternativa es considerar el código fuente del juego como la obra de arte. De esta manera el problema se reduce sustancialmente: lo que deberíamos ser capaces de conservar y reproducir es ese código fuente, creado, bien es cierto, con una tecnología de durabilidad cuestionable. Sin embargo, esto limita notablemente la riqueza de la obra, que solo adquiere pleno sentido cuando es puesta en ejecución por un jugador remoto.

En el mismo territorio de los juegos, Neil Hennessy desarrolló hace algunos años Jabber y Basho's Frogger mediante applets de Java. El primero de ellos, una generación aleatoria de palabras sin sentido, no depende del usuario, y en esta medida es más fácil de conservar. El segundo es un juego similar a los "comecocos" de moda hace algunos años, en el que el jugador es una rana que debe jugar una partida igualmente aleatoria. Como en el caso de los Arteroids de Jim Andrews, aquí tampoco es posible conservar las interacciones, que son las que proporcionan riqueza a la obra.

En el caso de Aya Karpinska, la autora ha desarrollado mediante applets de Java y flash poemas tridimensionales y sonoros en los que el usuario participa rotando a voluntad los espacios donde están escritos los versos, para leer estos de una manera o de otra. En algunos de sus poemas, como Poiesis, el usuario puede activar controles para escribir texto, incrementando de esta manera la experiencia poética. Evidentemente, como en los casos anteriores, si solo fuera posible conservar el código fuente se perdería el sentido de estas obras de arte, que consiste precisamente en las interacciones con el usuario.

Nick Montfort ha creado varios juegos, algunos de ellos basados en obras literarias, haciendo uso de Java. Estos juegos se pueden descargar en el equipo y se juega una versión fuera de línea con apariencia de diálogo en tiempo real. Si se juega en línea, el jugador dialoga, pregunta al programa acerca de cómo seguir jugando, con absoluta apariencia de estar dialogando con un sistema de inteligencia artificial. Además, si se introduce un URL conocido por varios jugadores, esta experiencia puede compartirse de manera real.

Fuera del territorio de los juegos, Ted Warnell ha desarrollado el programa Nari, que hace uso de XHTML y javascripts para devolver resultados aleatorios a partir de la introducción de un fichero, y permite al usuario remoto definir su configuración para generar sus propios poemas. En este caso, los poemas de Warnell son de fácil conservación, así como los creados por los usuarios remotos. Lo realmente problemático es conservar el programa, que es la obra, o una parte sustancial 
de ella — con independencia de las obras que se derivan de ella—, así como su documentación asociada.

Por último, Jason Freeman crea música digital en formato mp3 a partir de grabaciones analógicas, pero, sobre todo, ha desarrollado la aplicación iTunes Signature Maker, a partir del ejecutable iTunes de Apple, que establece un perfil del usuario y genera una firma basándose en el análisis automatizado de los ficheros de sonido que se encuentran en el equipo de este usuario.

\section{Continuidad y conservación}

De los ejemplos mencionados en la sección anterior esperamos que pueda deducirse que la conservación de creaciones digitales, en el sentido en el que se había entendido hasta ahora el concepto de conservación, no siempre es posible. El problema, como se adelantaba, es múltiple. En primer lugar, un objeto digital (y un objeto archivístico digital de manera mucho más dramática) nunca es simplemente un solo objeto digital. Tal y como Horsman escribía:

Para los (meta)datos archivísticos el objeto no siempre es un solo objeto discreto, sino a menudo un complejo de objetos interrelacionados, tanto físicos como abstractos, e incluidos documentos, agregaciones de documentos, creadores de documentos, procesos, conservadores, etc., reflejados en los tres principales conceptos archivísticos acerca de los documentos: contenido, estructura y contexto. Un documento de archivo no es un solo objeto de datos "discreto", y los datos acerca de él que los archiveros crean, recogen, actualizan, y utilizan no son solo metadatos descriptivos, sino que sirven a una diversidad de funciones archivísticas, incluidos la recuperación, la conservación, el almacenamiento, la evaluación, la disposición, etc. Son indispensables para el control físico, administrativo e intelectual sobre los materiales archivísticos. (Horsman, 2000)

Aunque Horsman llega a diferentes conclusiones, esta visión es similar a la planteada por el Proyecto InterPARES 1. En un entorno digital no es posible conservar el documento, sino solo la capacidad de reproducir el documento (Duranti, 2005b). El documento, como tal, es el conjunto de componentes digitales que se reúnen de nuevo cada vez que se les invoca, por ejemplo al hacer doble clic sobre un fichero de Word, con el objeto de rendir una representación adecuada y presumiblemente similar a la que se rindió la última vez que se invocó. Si esto es así en el contexto de un ordenador personal, en el de redes globales, con componentes dispersos en servidores repartidos por todo el mundo, la situación es mucho más compleja.

$\mathrm{Si}$ atendemos a los conceptos clave de la archivística — contenido, contexto y estructura-, los documentos digitales los tienen. No obstante, puede cuestionarse que sean interpretables del mismo modo que lo son en el entorno analógico. El contenido digital no es autoevidente: puede ser la reproducción de un solo fichero, o este fichero más el software o el plug-in que permiten su creación o reproducción (que serían, al mismo tiempo, elementos estructurales), o un conjunto de

Scire. 14 : 2 (jul.-dic. 2008) 65-85. ISSN 1135-3716. 
ficheros cuya reproducción depende de las interacciones con el usuario, etcétera. El contexto también es complejo: el contexto de creación puede consistir en la generación de una simple imagen estática o el escaneado de una fotografía, pero también en el desarrollo de un software propio, o de un software propio dependiente de componentes externos, más la documentación asociada, etcétera. La estructura, como se ha dicho, es un conjunto de componentes digitales físicamente separados dentro de un ordenador o a lo largo de toda la red global. Para complicarlo todo, las posibilidades de reproducir el contenido y de mantenerlo asociado a su contexto dependen de la estructura, que es de naturaleza tecnológica, en un momento en el que las tecnologías cambian y devienen obsoletas en plazos mucho más cortos que las necesidades de conservación de los documentos. Y nada se encuentra unido sobre un soporte físico estable. Por supuesto, todo tiene un carácter físico; de lo contrario, no sería un problema. Como ha indicado Duranti, lo que no es físico no perece y, por tanto, no hay que conservarlo (Duranti y Roeder, 2006). La cuestión es que los soportes físicos de los objetos digitales no son evidentes, están distribuidos y son inestables.

En estas circunstancias, no parece que la conservación, tal y como ha sido entendida por la teoría y la práctica archivísticas, siga resultando viable en todos los casos. Frente al concepto de conservación, quisiéramos sugerir el de continuidad; es decir, lejos de intentar conservar el contenido, el contexto y la estructura del documento, deberíamos intentar conservar el contenido en una forma que el creador reconozca como suya y que sea utilizable y comprensible para un usuario final, un contexto suficiente para explicar las circunstancias de creación, uso y continuidad del documento; y una estructura, original o no, que permita que el contenido y el contexto sigan siendo reproducibles y utilizables. En el actual estado de las nuevas tecnologías, las estructuras originales no tienen ninguna posibilidad de sobrevivir a largo plazo (ISO/TR 18492, 2005), pero si, por utilizar las mejores tecnologías disponibles, conservamos la capacidad de reproducir el contenido del documento y de mantenerlo enlazado a su contexto, habremos dado continuidad a la obra.

En la disciplina archivística, el término continuidad ha formado parte, tradicionalmente, del vocabulario cinematográfico. En sentido tradicional, continuidad es "el proceso de asegurar que los detalles de diferentes tomas se ajustan en el contexto de un programa" (Pearce-Moses, 2005). Sin embargo, Ben Howell Davis, en el marco de la puesta en valor de los activos digitales de una organización a efectos de maximizar su rentabilidad, ha recuperado el término en una acepción radicalmente diferente (Davis, 2005).

De acuerdo con el autor, la tecnología digital ha generado un espectro, al que llama el de la "complejidad acumulativa", que define a partir de estas tres aserciones: la complejidad se incrementa con la evolución, la complejidad engendra

Scire. 14 : 2 (jul.-dic. 2008) 65-85. ISSN 1135-3716. 
complejidad y la complejidad se incrementa con la conectividad. En el marco de los activos, no necesariamente económicos pero en el pensamiento del autor claramente orientados a la economía, de una organización, la complejidad acumulativa genera efímeros digitales crecientemente complejos y se deriva de tres condiciones enlazadas: la liquidez digital, o el modo en que los activos digitales se crean y utilizan; la continuidad digital, o el modo en que se almacenan los activos digitales y se accede a ellos; y la viabilidad digital, o el modo en que se mide el uso de los activos digitales. En el contexto que estamos considerando en el presente texto, la liquidez digital tiene su análogo en los procesos de creación y de uso/ interacción de la obra; la viabilidad digital tiene su análogo en el estudio económico que permitiría determinar si puede conservarse todo y, de no ser posible, qué debe conservarse. La continuidad digital tiene su análogo en lo que hasta este momento hemos llamado conservación. Puesto que este es el aspecto que nos ocupa, veamos el modo en que especifica Davis la continuidad digital:

La planificación estratégica para la conversión de formatos, la migración y la frecuencia de uso implica comprender e implantar normas, metadatos, formatos de almacenamiento y herramientas de búsqueda/acceso en evolución y que implican sofisticados vocabularios comunes. La atención a la continuidad digital asegura el uso a largo plazo de los activos. (Davis, 2005, p. 17)

Es decir, expandiendo la explicación de Davis, en un entorno crecientemente complejo y efímero, la única posibilidad de conservar es desarrollar estrategias de uso de las mejores tecnologías y normas disponibles, tecnologías y normas que, por lo demás, y en la medida en que ellas mismas se encuentran en evolución, no constituyen una garantía, sino meramente un incremento de las posibilidades. Implicada en esta permanente evolución de las normas y tecnologías se encuentra la aseveración ya sugerida de que no es posible conservar la estructura: nuevas tecnologías requerirán nuevas estructuras. No obstante, renunciar a conservar la estructura debería, a su vez, incrementar las posibilidades de dotar de continuidad a la reproducibilidad del documento y al enlace de este con su contexto, reflejado en metadatos.

\section{Conclusiones}

Esperamos haber mostrado que nos movemos en un entorno crecientemente complejo, de límites mal definidos y escasamente controlables. Este entorno está generando objetos digitales efímeros que tenemos la obligación de conservar, de manera radicalmente diferente a como se conservan los objetos analógicos. Aunque nadie conoce aún con claridad esta manera, existen esfuerzos de investigación, normalización y desarrollo que, al menos, intentan articular ciertas garantías. Cualquier actividad de conservación que pase por alto estos esfuerzos está destinada al fracaso, como lo está cualquiera que los respete. La diferencia estriba en el hecho

Scire. $14: 2$ (jul.-dic. 2008) 65-85. ISSN 1135-3716. 
de que en el segundo caso se obra con profesionalidad y se minimizan los riesgos, mientras que en el primero se obra con inconsciencia y se deja abierta la puerta a un caos que constantemente nos acecha. Nadie sabe si seremos capaces de conservar los objetos digitales que estamos creando como memoria para generaciones futuras, pero sí se sabe con bastante certidumbre que, de no renunciar al pensamiento analógico, de no investigar acerca de los mejores métodos, y de no aplicar los resultados de esta investigación, las generaciones futuras tendrán muchas cosas, salvo memoria.

\section{Notas}

(1) Tal es la definición proporcionada por el Proyecto InterPARES, que, por lo demás, recoge en su base de datos de terminología una cuidadosa selección de definiciones alternativas. Entre ellas: Documento creado o recibido y mantenido por una agencia, organización o individuo en seguimiento de obligaciones legales o en transacciones de negocio (http://www.archivists.org/glossary/index.asp). Datos o información que ha sido fijada a algún soporte; que tiene contenido, contexto y estructura; y que se utiliza como una extensión de la memoria o la responsabilidad humanas. (http://www.archivists.org/ glossary/index.asp). Documentos realizados o recibidos por autoridades públicas en el curso de ejecución de su función pública. (http://www.slais.ubc.ca/RESOURCES/ students/Archival_Terminology.pdf). Una representación de un hecho o acto que está memorado en un portador físico — esto es, un soporte - y conservado por una persona física o jurídica en el curso de ejecución de sus actividades (http://www.interpares.org/ book/index.htm). Información registrada producida o recibida en el inicio, desarrollo o finalización de una actividad institucional o individual, que comprende contenido, contexto y estructura suficientes para proporcionar evidencia de la actividad. Documento(s) producidos o recibidos por una persona u organización en el curso de sus actividades, y retenido(s) por esa persona u organización. Un documento (record) puede incorporar uno o varios documentos (documents) (por ejemplo, si un documento tiene adjuntos), y puede estar sobre cualquier soporte en cualquier formato. Además del contenido del (de los) documento(s), debiera incluir información contextual y, si es aplicable, información estructural (i. e., información que describe los componentes del documento). Una característica clave del documento es que no puede cambiarse (http://www.cornwell.co.uk/moreq.html). Los documentos constituyen un registro auténtico de los procedimientos ante un tribunal, incluyendo la demanda u otro proceso generador, las declaraciones y la sentencia, pero usualmente no la evidencia presentada (http://www.oxfordreference.com/views/ENTRY.html?subview=Main\&entry=t49.e3046). Información (documentos) registrada con independencia de la forma o medio de creación, recibida y mantenida por una agencia, institución, organización o individuo en seguimiento de sus obligaciones legales o en las transacciones de negocio. En procesamiento de datos, una agrupación de elementos interrelacionados que forman la unidad básica de un fichero (http://www.archivists.org/glossary/index.asp).

(2) Cf., simplemente a modo de breve ejemplo, Shirkey, Cynthia D. E-poetry: Digital frontiers for an evolving art form. // College \& Research Libraries News. 4:64 (April 2003). El conciso listado de corrientes en poesía electrónica no es sino una muestra de las in- 
finitas concepciones y definiciones que los artistas proporcionan de su trabajo. http://www.ala.org/ala/acrl/acrlpubs/crlnews/backissues2003/april1/epoetry.htm (200606-11). Pueden confrontarse distintas poéticas específicas, y orientadas tanto al hipertexto como a los multimedia, en portales como E-Poetry: Electronic Poetry Center (http://epc.buffalo.edu/e-poetry/) (2006-06-11), Ubuweb (http://www.ubu.com/) (2006-06-11) o la revista Dichtung-Digital (http://www.dichtung-digital.de/) (2006-06-11).

(3) Desde un punto de vista conceptual, este argumento no es totalmente honesto. Es decir, conceptualmente no existe diferencia entre un creador que es un artista y un creador que es una organización con responsabilidades sociales, jurídicas o administrativas. Los criterios que se apliquen al artista deberían ser los mismos que se aplicaran a la organización. Sin embargo, desde un punto de vista práctico es concebible la argumentación de que, a efectos de responsabilidad social, la pérdida de la partitura y de todas las representaciones o ejecuciones de La flauta mágica supondría un daño irreparable para el patrimonio cultural de la humanidad; sin embargo, la humanidad sobreviviría a esto. La pérdida de los documentos que han permitido desencadenar la lucha contra la corrupción en el Ayuntamiento de Marbella, o de los que han hecho posible demostrar que en Irak no existían armas químicas y que George Bush ha llegado a unos límites de irresponsabilidad social difícilmente superables implicaría un daño irreparable para la memoria ética de la humanidad. La humanidad sobreviviría a esto, pero de forma menos responsable. Digamos que podemos permitirnos el lujo de perder La flauta mágica, pero, después de algunos miles de años de existencia poco ética, no podemos permitirnos el lujo de perder la poca responsabilidad social que nos queda, y que queda garantizada por el reflejo de las acciones en documentos.

(4) Podría argumentarse con solidez que este problema ya existe en el entorno analógico. Por ejemplo, a un libro de actas deteriorado se le somete a procesos físicos y químicos que alteran su estructura pero permiten su conservación. No obstante, estos procesos no alteran el contenido del libro de actas. En el caso de un documento digital, el contenido y la forma en que se reproduce de manera legible por humanos son altamente dependientes de la estructura, los componentes digitales, etcétera. Si se altera la estructura, y esto parece inevitable en el actual entorno tecnológico, si es que se quiere intentar asegurar la permanencia del contenido y del contexto, estos, paradójicamente, quedan comprometidos. Nunca antes había sido tan cierto que el medio es el mensaje.

\section{Referencias}

Andrews, Jim. Animisms. Vispo.com, 1995-2006. http://www.vispo.com/animisms/index.html (2007-01-28).

Andrews, Jim. Arteroids. http://www.vispo.com/arteroids/index.htm (2007-01-28).

Andrews, Jim. Vispo: Langu(im)age. http://www.vispo.com/ (2007-01-28).

Andrews, Jim. Word For/Word: a Journal of New Writing. http://www.wordforword.info/ vol9/index2.htm (2007-01-28).

Davis, Ben Howell (2005). Cumulative complexity: understanding the scope of digital asset liquidity, continuity, and viability. // Journal of Digital Asset Management. 1:1 (2005) 16-24.

Scire. 14 : 2 (jul.-dic. 2008) 65-85. ISSN 1135-3716. 
Delgado Gómez, Alejandro (2005). Archivos y metadatos de conservación: estado del arte y propuesta metodológica. // Scire. 11:1 (2005) 83-101.

Digital Longevity. http://www.digitaleduurzaamheid.nl/index.cfm?paginakeuze=286 (200701-28).

Duranti, Luciana (2004a). Conservar arte electrónico auténtico a largo plazo: el Proyecto InterPARES 2 (presentado en el Congreso Anual del Electronic Media Group del American Institute for Conservation of Historic and Artistic Works en Portland, Oregón, en 2004).

Duranti, Luciana (2004b). La questione fondamentale: in quali entità digitali si concreta la memoria del futuro? Turín, 2004.

Duranti, Luciana (2005a). Expected outcomes of InterPARES 2. // InterPARES, 2005 (acceso restringido).

Duranti, Luciana (ed.) (2005b). La conservación a largo plazo de documentos electrónicos auténticos: hallazgos del Proyecto InterPARES. Cartagena: Ayuntamiento / 3000 Informática, 2005.

Duranti, Luciana; Roeder, John (2006). La conservazione a lungo termine dell'autenticitá di composizioni digitali interattive: la ricerca di InterPARES 2. Archivi \& Computer. (2006) 35-42.

Encyclopaedia Britannica Online. http://www.britannica.com/ebc/article-9355856?query= art\&ct $=(2007-01-28)$.

E-Poetry: Electronic Poetry Center. Buffalo University, 2006. http://epc.buffalo.edu/ (200701-28).

Expertisecentrum David (eDavid). http://www.expertisecentrumdavid.be/eng/index.php (2007-01-28).

Freeman, Jason. http://www.jasonfreeman.net/itsm/ (2007-01-28).

Gilliland-Swetland, Anne (2005). Discussion paper on the nature and role of metadata in the creation of reliable and the preservation of authentic records in electronic systems. InterPARES, 2005.

Harvey, Ross (2005). Preserving digital materials. Múnich: K. G. Saur, 2005.

Hennessy, Neil. Basho's Frogger. http://epc.buffalo.edu/authors/hennessey/data/ basho_frogger/index.html (2007-01-28).

Hennessy, Neil. Jaber: The Jabberwocky Engine. http://epc.buffalo.edu/authors/hennessey/ data/jabber/index.html (2007-01-28).

Horsman, Meter (2999). Metadata and archival description. EUAN, 2000. http://www.euan.org/ euan_meta.html (2007-01-28).

The Humanities Advanced Technology and Information Institute (HATII), University of Glasgow; The National Initiative for a Networked Cultural Heritage (NINCH). The $\mathrm{NINCH}$ guide to good practice in the digital representation and management of cultural heritage materials, 2002. http://www.nyu.edu/its/humanities/ninchguide/ (2007-01-28).

InterPARES 2 Project. http://www.interpares.org/rws/rws_terminology.cfm?letter=d (200701-28).

ISO/DIS 22938 (2006). Document management - Electronic content/document management data interchange format. International Organization for Standardization, 2006.

Scire. 14 : 2 (jul.-dic. 2008) 65-85. ISSN 1135-3716. 
ISO/TR 18492 (2005). Long-term preservation of electronic document-based information. International Organization for Standardization, 2005.

Karpinska, Aya. Poiesis. http://www.technekai.com/poiesis/poiesis.html (2007-01-28).

Lialina, Olia. My boyfriend came back from the war. http://myboyfriendcamebackfromth. ewar.ru/ (2007-01-28).

McKemmish, Sue (1996). Evidence of me... // Archives and Manuscripts. 24:1 (1996). http://www.sims.monash.edu.au/research/rcrg/publications/recordscontinuum/smckp1.html (2007-01-28).

Merriam-Webster Online Dictionary. http://www.m-w.com/cgi-bin/dictionary (2007-01-28).

Minerva Knowledge Base. Roma: Ministero per i Beni e le Attività Culturali, Istituto Centrale per il Catologo Unico delle Biblioteche e per le Informazioni Bibliografiche, 2006. http://www.minervaeurope.org/ (2007-01-28).

Montfort, Nick. http://nickm.com/ (2007-01-28).

Pearce-Moses, Richard (2005). A glossary of archival and records terminology. http://www.archivists.org/glossary/term_details.asp?DefinitionKey=78 (2007-01-28).

PREMIS (PREservation Metadata: Implementation Strategies) (2002). http://www.oclc.org/ research/projects/pmwg/ (2007-01-28).

Real Academia Española. Diccionario de la Lengua Española. http://www.rae.es/ (2007-01-28).

Rhizome. New York: New Museum, 1996-. http://www.rhizome.org/ (2007-01-28).

ERPANET, Selecting Technologies Tool (2003). http://www.erpanet.org/guidance/docs/ ERPANETSelect_Techno.pdf (2007-01-28)..

Thibodeau, Kenneth (2002). Overview of technological approaches to digital preservation and challenges in coming years. // The state of digital preservation: an international perspective. Council on Library and Information Resources, 2002. http://www.clir.org/pubs/ reports/pub107/thibodeau.html (2007-01-28)..

Ubuweb. Ubuweb Foundation, 2006. http://www.ubu.com/ (2007-01-28)..

Uribe, Ana María. Tipoemas y anipoemas. Vispo.com, 1997-2003. http://www.vispo.com/ uribe/index.html (2007-01-28).

Vogt-O'Connor, Diane; Paul Conway (2002). Handbook for digital projects: a management tool for preservation and access. http://www.nedcc.org/digital/dighome.htm (2007-01-28).

Warnell, Ted. Poem by Nari. http://warnell.com/windows/nari.htm (2007-01-28).

Scire. $14: 2$ (jul.-dic. 2008) 65-85. ISSN 1135-3716. 